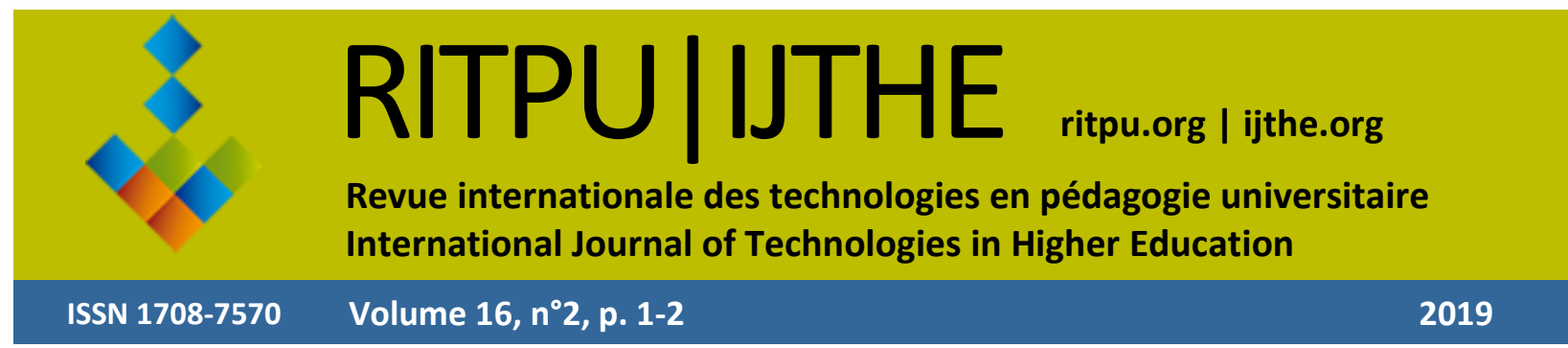

\section{Du plagiat à l'intégrité académique : quelles compétences, quelles stratégies? Introduction au numéro thématique}

https://doi.org/10.18162/ritpu-2019-v16n2-01
Sylvie GERVAIS,

Université du Québec en Outaouais

Canada

Sylvie.gervais@uqo.ca

Martine PETERS

Université du Québec en Outaouais

Canada

martine.peters@uqo.ca

Il est désormais avéré que les étudiants consultent fréquemment le Web pour rechercher de l'information. Avec le Web et son abondance d'information s'est également répandu le copier/coller, transformant notamment les pratiques d'écriture.

À la manière du scrapbooking, le créacollage numérique consiste à trouver, sélectionner, rassembler et organiser l'information provenant du Web, qu'elle soit sous forme de textes, d'images, de vidéos ou de sons, afin de réaliser une toute nouvelle création écrite (Peters, 2015). L'ensemble de ces actions dont le but ultime est l'écriture ont recours à des stratégies de créacollage numérique $(\mathrm{SCN})$ qui font appel à des compétences informationnelles, rédactionnelles et de référencement documentaire.

Afin de s'assurer que les étudiants possèdent ces compétences et qu'ils développent de bonnes pratiques de créacollage numérique, les enseignants doivent les former différemment et adéquatement. L'intégrité dans les études figure parmi les enjeux auxquels doit répondre la formation scolaire et universitaire des élèves et des étudiants d'aujourd'hui.

Ce numéro thématique propose donc un regard sur les stratégies de créacollage numérique et les compétences qu'elles mobilisent ainsi que sur l'intégrité dans les études afin de prémunir les étudiants contre le plagiat.

Dans Les stratégies d'écriture universitaire numérique : pratiques déclarées d'étudiants et d'enseignants québécois (p. 5), Vincent, Fontaine, Peters et Boies proposent de définir la nature des stratégies de créacollage numérique touchant les compétences scripturales puis de vérifier l'influence du sentiment d'efficacité personnel en lecture et en écriture pour finalement déterminer quelles sont les stratégies envisagées par les enseignants.

Deux textes abordent les compétences de référencement documentaire : celui de Cadieux, Peters et Beauchemin-Roy, Étude de compétences de référencement documentaire d'étudiants universitaires (p. 24), et celui de Monney, Peters, Boies et Raymond, Évaluer la compétence 
de référencement documentaire chez des étudiants de premier cycle universitaire : pratiques déclarées d'enseignants universitaires (p. 39).

L'étude de Cadieux, Peters et Beauchemin-Roy explore les liens entre savoirs, savoir-faire et savoir-être et le nombre d'années universitaires. Afin de tracer les compétences de référencement documentaire des étudiants tout au cours de leur parcours universitaire, les auteurs prennent en considération trois dimensions: "l'utilisation de logiciels de référencement, l'efficacité personnelle en référencement et la résolution de problèmes en matière de référencement ».

Pour leur part, Monney, Peters, Boies et Raymond soulèvent la question de l'enseignement et de l'évaluation de la compétence de référencement documentaire afin de répondre à trois objectifs : «déterminer les cibles d'apprentissage définies par les professeurs pour développer la compétence de référencement, dégager les pratiques de rétroaction mises en place par les enseignants, cerner les pratiques d'évaluation formelle de la compétence de référencement documentaire chez les étudiants ». Leurs résultats indiquent que la compétence de référencement documentaire n'est pas forcément vue comme une cible d'apprentissage par les enseignants et que cette compétence est évaluée avec indulgence.

Le numéro thématique se termine par trois textes sur le plagiat: Qu'est-ce qui détermine les étudiants à plagier: l'infrastructure informatique de Google ordinaire ou la « commodité »? (p. 56) de Boubée, suivi de Rethinking the relationship between plagiarism and academic integrity (p. 69) de Jamieson et Moore Howard, puis de Stopping plagiarism through enculturation: A practice-based approach de McDonald et Adl (p. 86).

Dans Qu'est-ce qui détermine les étudiants à plagier? Boubée s'interroge d'une part sur les usages de Google par les étudiants, puis sur les caractéristiques de l'infrastructure informatique et informationnelle du moteur de recherche. Selon l'auteure, «deux activités sont cruciales du point de vue du plagiat : l'exploration de la littérature et la différenciation du statut des sources ». L'objectif est d'associer deux dimensions, technologie et activité informationnelle, pour mieux saisir les liens entre plagiat universitaire et recherche d'information.

Jamieson et Moore Howard, dans Rethinking the relationship between plagiarism and academic integrity, retracent l'évolution et les différentes définitions du concept d'intégrité dans les études depuis les années 1960. Une analyse des erreurs textuelles produites par les étudiants amène les auteures à regrouper sous le parapluie de l'intégrité des études les différentes formes de tricherie, privilégiant ce seul et même concept. Jamieson et Moore Howard soutiennent également que la pédagogie, et non la punition, est la solution aux copier/coller et aux erreurs de citation.

Finalement, McDonald et Adl analysent dans Stopping plagiarism through enculturation, les effets de la méthode ALTE (Academic Literacies Training in English) pour favoriser le développement des compétences de citation des étudiants. Dans une perspective d'enculturation, les auteurs cherchent à transmettre aux étudiants des valeurs universitaires, particulièrement la propriété intellectuelle. Selon eux, la méthode ALTE, qui adopte une approche fondée sur la pratique, est utile pour réduire le plagiat par inadvertance.

\section{Références}

Peters, M. (2015). Enseigner les stratégies de créacollage numérique pour éviter le plagiat au secondaire. Revue canadienne d'éducation, 38(3). Récupéré de http://journals.sfu.ca/cje 